\title{
Star formation rates, laws, thresholds
}

\author{
Stefanie Walch \\ I. Physics Institute, University of Cologne \\ Zülpicher Str. 77, 50937 Cologne \\ email: walch@ph1.uni-koeln.de
}

\begin{abstract}
On scales of $\gtrsim$ several hundred parsec, there are relatively well defined observed relations between the star formation rate surface density of a galaxy and its gas content. I present a short summary of the so-called 'star formation laws' and discuss the most established models to explain their origin. Nevertheless, none of the models can explain the offset of these relations, which requires a low star formation efficiency within the total and/or molecular gas. Stellar feedback is proposed to limit the star formation efficiency locally. I discuss the available feedback mechanisms and show recent simulation results on the evolution of the multi-phase interstellar medium under the influence of gravity, cooling, feedback-driven outflows and heating.
\end{abstract}

Keywords. stars: formation, ISM: supernova remnants, ISM: general, ISM: kinematics and dynamics, ISM: structure, turbulence, MHD, galaxies: ISM

\section{The galactic 'star formation law'}

The most established observed 'star formation (SF) law' in galaxies is the KennicuttSchmidt relation (Schmidt, 1959; Kennicutt, 1989; Kennicutt, 1998), which relates the average star formation rate $(\mathrm{SFR})$ surface density $\left(\Sigma_{\mathrm{SFR}}\right.$ in units of $\left.\left[\mathrm{M}_{\odot} \mathrm{yr}^{-1} \mathrm{kpc}^{-2}\right]\right)$ to the total gas surface density $\left(\Sigma_{\mathrm{G} \text { as }}\right.$ in units of $\left.\left[\mathrm{M}_{\odot} \mathrm{kpc}^{-2}\right]\right)$ of a galaxy using a power law $\Sigma_{\mathrm{SFR}} \propto \Sigma_{\mathrm{Gas}}^{n_{\mathrm{KS}}}$. Schmidt (1959) originally suggested $n_{\mathrm{KS}} \approx 2$, while Kennicutt (1998) determines $n_{\mathrm{KS}} \approx 1.4$. A power law index of $n_{\mathrm{KS}}=1.5$ can be readily explained with a dynamical model of star formation on galactic scales. This can be formulated in two ways: intrinsically three-dimensional - per free-fall time of the denser, star-forming molecular clouds, $\tau_{\mathrm{ff}} \propto\left(G \rho_{\mathrm{G} \text { as }}\right)^{-1 / 2}$; or intrinsically two-dimensional - per galaxy dynamical time, $\tau_{\mathrm{dyn}}$, which can be calculated from the Toomre criterion (Elmegreen-Silk relation; Elmegreen, 1997; Silk, 1997; see e.g. Kennicutt, 1998, and Genzel et al., 2015 for an application). The first argument goes as follows (e.g. Krumholz et al., 2005, Krumholz \& Tan, 2007, Leroy et al., 2008):

$$
\Sigma_{\mathrm{SFR}}=\epsilon_{\mathrm{SF}} \Sigma_{\mathrm{Gas}} \times \text { dynamical rate, }
$$

where the dynamical rate is $1 / \tau_{\mathrm{ff}}$ in this case, and $\epsilon_{\mathrm{SF}}$ is the star formation efficiency. Assuming a constant gas scale height we have $\Sigma_{\mathrm{G} \text { as }} \propto \rho_{\mathrm{G} \text { as }}$ and therefore $n_{\mathrm{K}}=1.5$. These global dependencies have been verified in numerous numerical studies that mostly include simple hydrodynamics and gas cooling (e.g. Tasker et al., 2006; Harfst et al., 2006; Wada \& Norman, 2007).

However, a constant scale height is not a good assumption for all star-forming galaxies (e.g. in dIrr's, where $H \propto 1 / \Sigma_{\mathrm{G} \text { as }}$ with scale height $H$, which implies $\rho \propto \Sigma_{\mathrm{G} \text { as }}^{2}$; Elmegreen \& Hunter, 2015). Therefore, the simple Toomre analysis fails in these galaxies while models based on the volumetric density (e.g. Krumholz \& Matzner, 2009) work well. In addition, in radially resolved galaxies the observed slopes seem to vary with galactocentric radius, from linear in the inner discs to quadratic in the outer discs (Barnes 
et al.2012). Elmegreen (2015) propose disc flaring as a theoretical explanation for a steeper SF relation in outer discs.

Interestingly, the gas depletion time, $\tau_{\text {dep }}$, in a normal star forming galaxy is quite long, around 2 Gyr (Genzel et al. 2010). This implies that the star formation efficiency in this model is low with $\epsilon_{\mathrm{SF}}=\tau_{\text {ff }} / \tau_{\text {dep }} \sim 1-3 \%$. The question remains why star formation is so inefficient. This might be caused by super-sonic turbulence that prevails within molecular clouds and shapes their sub-structure into a log-normal density PDF (Vazquez-Semadeni, 1994; Padoan et al., 1997; Padoan \& Nordlund, 2002). Since the free-fall time is density dependent, only the densest parts of the clouds would undergo gravitational collapse (see e.g. Krumholz et al., 2005), which lowers the overall SF efficiency (for numerical tests see e.g. Federrath \& Klessen,2013).

The 'molecular star formation law'.

More recently, Bigiel et al. (2008) and Leroy et al. (2008) could show that there is a tight and almost linear correlation of $\Sigma_{\mathrm{SFR}}$ with the molecular gas surface density $\Sigma_{\mathrm{Mol.Gas}}$, which is inferred from $\mathrm{CO}$ observations using a CO-to- $\mathrm{H}_{2}$ conversion factor, $\alpha_{\mathrm{CO}}$. The linear relation is still under debate. Shetty et al. (2014a, 2014b) carry out a Bayesian analysis of the Bigiel et al. (2008) sample and report sub-linear slopes, while e.g. Momose et al. (2013) report super-linear slopes. In Fig. 1, we compile all popular scaling relations of $\Sigma_{\mathrm{SFR}} \propto \Sigma_{\mathrm{Gas}}$ and $\Sigma_{\mathrm{SFR}} \propto \Sigma_{\mathrm{Mol.Gas}}$, which have been inferred from observations.

To understand the molecular KS relation, one has to additionally consider the transition from atomic to molecular gas. Krumholz et al. (2008) determine the atomic-tomolecular transition for idealised, spherical gas clouds under external radiation. Sternberg et al. (2014) present an alternative derivation, which leads to a similar formulation for the molecular-to-atomic gas ratio as a function of metallicity and external radiation field. These models predict a metallicity-dependent transition from atomic to predominantly molecular gas at $\Sigma_{\mathrm{G} \text { as }} \approx 10 \mathrm{M}_{\odot} \mathrm{pc}^{-2}$ (for solar metallicity) and a steep decline in molecular gas fraction for lower $\Sigma_{\mathrm{G} \text { as }}$. This is consistent with observations of resolved disc galaxies e.g. by Schruba et al. (2011).

Here it is worth pointing out that molecules, although their presence is tightly connected with star formation, seem to be a companion (forming at high density and low temperature) rather than the cause of star formation. The contribution of molecular cooling to the total cooling rate is negligible (Glover \& Clark, 2012). Dust shielding seems to be the dominant physical process to allow gas cooling and star formation. Recently, Clark \& Glover (2014) report that dust shielding naturally explains the apparent star formation threshold at $\Sigma_{\mathrm{G} \text { as }} \approx 120 \mathrm{M}_{\odot} \mathrm{pc}^{-2}$ (Evans et al., 2009; Lada et al., 2010; Heiderman et al., 2010; Lada et al., 2012). Alternatively, Burkert \& Hartmann (2013) show that this surface density could just coincide with the typical density at which self-gravity becomes dominant.

In any case, the star formation efficiency, i.e. the offset of the molecular KS relation, is still low, $\epsilon_{\mathrm{SF}} \sim 1-3 \%$ and thus, the formation of dense molecular clouds seems not to be the rate limiting step in the star formation process. Feedback from massive stars on molecular cloud scales is still required to moderate $\epsilon_{\mathrm{SF}}$.

Scale dependence.

With increasing spatial resolution, the notion of a constant depletion time scale gradually breaks down. Onodera et al. (2010) and Schruba et al. (2010) show that this happens at scales below $\sim 200$ pc. On smaller scales, where one would either center on peaks in the molecular gas distribution to measure $\Sigma_{\mathrm{Mol.G} \text { as }}$ or on luminous star forming regions, the spatial correlation between the two seems to diverge and the KS relation, respectively the molecular KS relation, fails on molecular cloud scales (Kruijssen et al. 2014). 


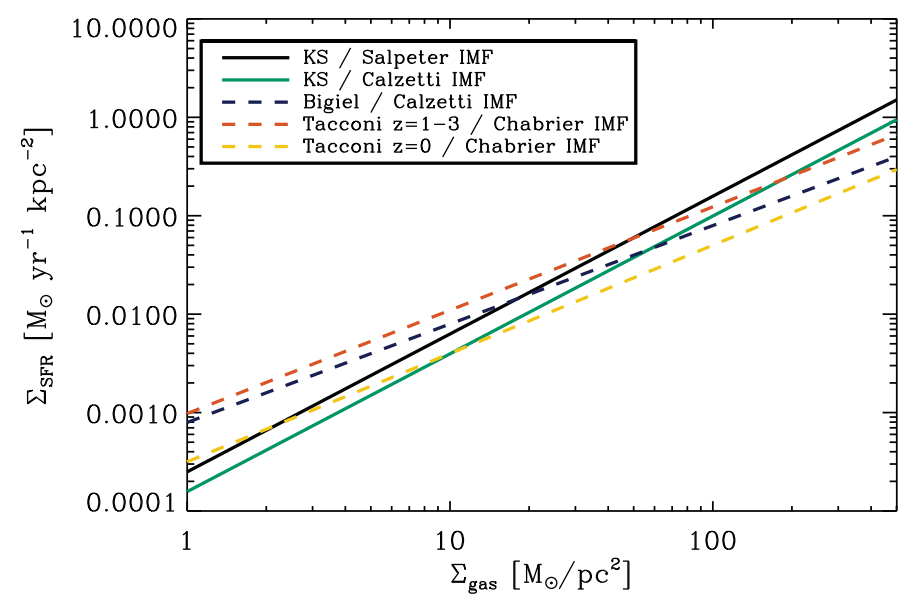

Figure 1. A compilation of proposed power-law scaling relations of the SFR surface density, $\Sigma_{\mathrm{SFR}}$, vs. total gas surface density, $\Sigma_{\mathrm{G} \text { as }}$ (full lines), or molecular mass surface density, $\Sigma_{\mathrm{Mol.Gas}}$ (dashed lines), respectively. The scaling relations of $\Sigma_{\mathrm{SFR}}$ vs. $\Sigma_{\mathrm{G} \text { as }}$ follow a power law index of 1.4. The offset depends on the underlying stellar IMF. Here we show two possibilities: (1)Black line: the standard Kennicutt-Schmidt (KS) relation (Kennicutt, 1998) as derived for a Salpeter IMF (Salpeter, 1955), and (2) green line: the KS relation scaled with the IMF from Calzetti et al. 2007, which is the standard IMF in STARBURST99 (Leitherer et al.,1999). The scaling relations of $\Sigma_{\mathrm{SFR}}$ vs. $\Sigma_{\mathrm{Mol.G}}$ are approximately linear. We show the results of (3) blue line: Bigiel et al. (2008), who use the same IMF as (2); (4) red line: Tacconi et al. (2013), their fit to high redshift galaxies, and (5) yellow line: Tacconi et al. (2013), their fit to low redshift galaxies, both of which assume a Chabrier IMF (Chabrier, 2001). We note that $\Sigma_{\text {Mol.G as }}$ should approach $\Sigma_{\mathrm{G} \text { as }}$ for high gas surface densities, where basically all gas is in molecular form. Figure taken from Walch et al. (2015). (A color version is available online.)

The local star formation efficiency per free-fall time seems to be enhanced to $\sim 10 \%$ on molecular cloud scales (Lada et al., 2010; Murray, 2011; Evans, 2014), which implies shorter gas depletion times of $10-100 \mathrm{Myr}$ (see Agertz et al., 2015 for an overview plot).

\section{Is feedback regulating star formation on galactic scales?}

What is the cause of the low star formation efficiency in galaxies and in molecular clouds? Since it has been recognised that molecular clouds are probably not long-lived objects but are governed by supersonic turbulence and evolve on a dynamical time scale (see e.g. Pringle et al., 2001), a stabilisation of the clouds against gravitational collapse by e.g. magnetic fields is most likely not a viable explanation for the low observed star formation efficiencies. In addition, turbulence decays on a crossing time (Stone et al. , 1998; MacLow et al. , 1998) and needs to be continuously replenished. Stellar feedback in its different forms, i.e. ionizing radiation and radiation pressure, stellar winds and Supernova explosions, is a viable mechanism to quench star formation in molecular clouds by unbinding gas, to drive turbulence within the interstellar medium (ISM), as well as to drive galactic fountains and outflows.

In 2010, Ostriker et al. developed a model of self-regulated star formation in galactic discs. Assuming that the intensity of ionizing radiation scales linearly with $\Sigma_{\mathrm{SFR}}$ and that the star formation rate is proportional to the mass in the self-gravitating component, they derive a model in which the thermal (based on UV heating and cooling) and vertical dynamical equilibria are simultaneously fulfilled by matching the thermal (of the warm, diffuse phase) and mid-plane gas pressures. The model predicts that $\Sigma_{\mathrm{SFR}} \propto \Sigma_{\mathrm{Gas}} \sqrt{\rho_{\mathrm{star}+\mathrm{DM}}}$, where $\rho_{\text {stars D M }}$ is the mid-plane density of stars plus dark matter. Star formation then 


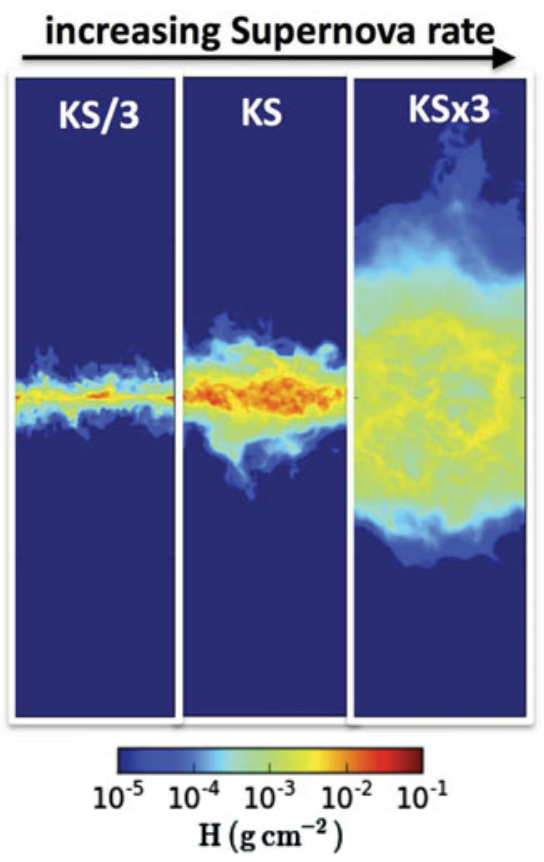

Figure 2. Column density distribution of atomic hydrogen as seen edge on for SILCC simulations including Supernova feedback which is correlated with dense gas (peak driving). Left to right: with increasing the Supernova rate from $\frac{1}{3} \times$ the typical $\mathrm{KS}$ value to $3 \times$ the typical $\mathrm{KS}$ value the disc scale height increases. See Walch et al. (2015) and Girichidis et al. (2016) for further information. (A color version of this figure is available online.)

self-regulates, since high SFRs lead to an increase in mid-plane pressure, which causes a decrease in mid-plane density and thus, a decrease in SFR, leading to a decrease in mid-plane pressure etc. The model works well for many different galaxies. In particular, it is able to explain the observed star formation rates in HI dominated outer discs (Roychowdhury et al., 2015) as it predicts - due to the decrease of thermal equilibrium pressure in the outer disc - that star formation can still occur in low density gas in the outer discs rather than having a sharp cut-off radius as predicted by models which tightly couple the SFR to the molecular gas fraction.

Supernova feedback.

Energetically, it seems that Supernovae are the most important feedback mechanism (e.g. Agertz et al., 2013). However, considering that they only explode after a few Myr, when the first massive stars reach the end of their life times, their role for quenching or triggering star formation within individual MCs is still unclear and needs to be investigated in numerical studies. Furthermore, Supernovae cause the formation of the observed hot, volume-filling gas phase (McKee \& Ostriker, 1977).

In a series of papers, Kim \& Ostriker (e.g. 2011, 2013, 2015) investigate the selfregulation by feedback from massive stars, here in the form of type II Supernovae, that are correlated with the dense, star-forming gas and explode instantaneously to quench local collapse. These simulations quickly establish a dynamical equilibrium in the ISM, where gravitational collapse is balanced by turbulent and thermal pressure. They recover the scaling relation of Ostriker et al. (2010).

In the recent work by Gatto et al. (2015) the evolution of the Supernova-driven multiphase ISM is studied in 3D simulations with the AMR code FLASH (Fryxell et al., 2000). We use a periodic box of size $(256 \mathrm{pc})^{3}$ with different initial densities, and compare three different driving schemes for the Supernovae - peak driving (all Supernovae are associated with density peaks), random driving (all Supernovae explode in random positions), and mixed driving (mix of peak and random driving). The results clearly show that peak driving leads to an ISM which is too cold (very low hot gas fractions) and therefore not 
in agreement with observations. Random driving on the other hand, leads to an ISM that is too hot: Supernovae primarily explode in low density gas which becomes hotter and hotter and compresses most of the mass into dense, molecular clumps. Here we are limited by the numerical setup, which doesn't allow the gas to escape in the vertical direction as would happen in a stratified disc that would develop a galactic outflow.

The SILCC project: Supernova-driven ISM in stratified discs.

In the SILCC project (SImulating the Life-Cycle of molecular Clouds; Walch et al., 2015; Girichidis et al., 2016), we study the Supernova-driven, multi-phase ISM in a stratified galactic disc including chemistry, cooling, and self-gravity. We choose solar neighbourhood conditions (gas surface density of $\Sigma_{\text {gas }}=10 \mathrm{M}_{\odot} \mathrm{yr}^{-1}$ ) and solar metallicity and explore the effect of different Supernova rates $\left(\frac{1}{3}, 1\right.$, and $3 \times$ the rate expected from a star formation rate according to the KS relation and a massive star fraction in agreement with the Chabrier IMF; Chabrier, 2001). We find that peak driving leads to a dynamical equilibrium in the ISM, but at the same time, the ISM is lacking a hot component (since SNe in dense gas cool quickly) as well as a molecular component (since molecular gas is heated and dispersed by the feedback). In Fig. 2 we show the column density of atomic hydrogen for runs with peak driving at different Supernova rates at $t=50 \mathrm{Myr}$. The disc scale height increases with increasing Supernova rate, but no galactic fountains or outflows are driven with peak driving. Instead, a random Supernova component is required in order to establish a hot, volume-filling phase and drive outflows. The downside is that - unlike in simulations without self-gravity (e.g. Joung \& MacLow, 2006; Hill et al. 2012; Gent et al. 2013) - there is no dynamical equilibrium established in simulations with self-gravity and a significant fraction of random Supernovae, as the dense molecular clouds cannot be disrupted. In addition, the delay time of Supernovae in dense regions (5-40 Myr, depending on the mass of the progenitor) renders them inefficient in dispersing dense gas and regulating star formation. Therefore the inclusion of other feedback processes (ionizing radiation, stellar winds, radiation pressure) is required to regulate star formation.

Other feedback processes.

Ionizing radiation can disperse typical, small molecular clouds with $M \approx 10^{4} \mathrm{M}_{\odot}$ in a few Myr (Walch et al., 2012). In Walch et al. (2012), we used a new approach to describe the initial MC structure with a clumps-within-clumps algorithm, which is parameterized in terms of a fractal dimension, $D$. The fractal dimension $D$ describes the clumpiness of the underlying MC structure. We use the method of Bisbas et al. (2009) to model the expansion of HII regions in these clouds and we were able to show that the cloud dispersal is independent of $D$. This finding is supported by other authors (e.g. Dale et al., 2012). In particular, Dale et al. (2012) show that the cloud escape velocity, $v_{\text {esc }}$, is the critical parameter which determines the susceptibility of a cloud to ionizing radiation, where clouds with $v_{\text {esc }} \lesssim 10 \mathrm{~km} / \mathrm{s}$ can be heavily affected. This implies that more massive clouds with $M \approx 10^{5 \ldots 6} M_{\odot}$ are affected by ionzing radiation, but the expansion of the HII region would stall before it reaches the edge of the cloud and drives outflows. Therefore, additional feedback like e.g. Supernovae is required to disperse more massive clouds. Analytically, the impact of different feedback mechanisms on molecular clouds (MCs) has been estimated by Matzner et al. (2002), Fall et al. (2011), and Murray et al. (2010). The latter two works stress that radiation pressure could be important in massive MCs (see also Krumholz \& Matzner, 2009). Due to space limitations I refrain from further discussing radiation pressure here.

Even before the Wolf-Rayet phase, massive stars have typical mass loss rates of $10^{-6} \mathrm{M}_{\odot} \mathrm{yr}^{-1}$ and drive a wind with a velocity of a few $1000 \mathrm{~km} \mathrm{~s}^{-1}$. Therefore they 

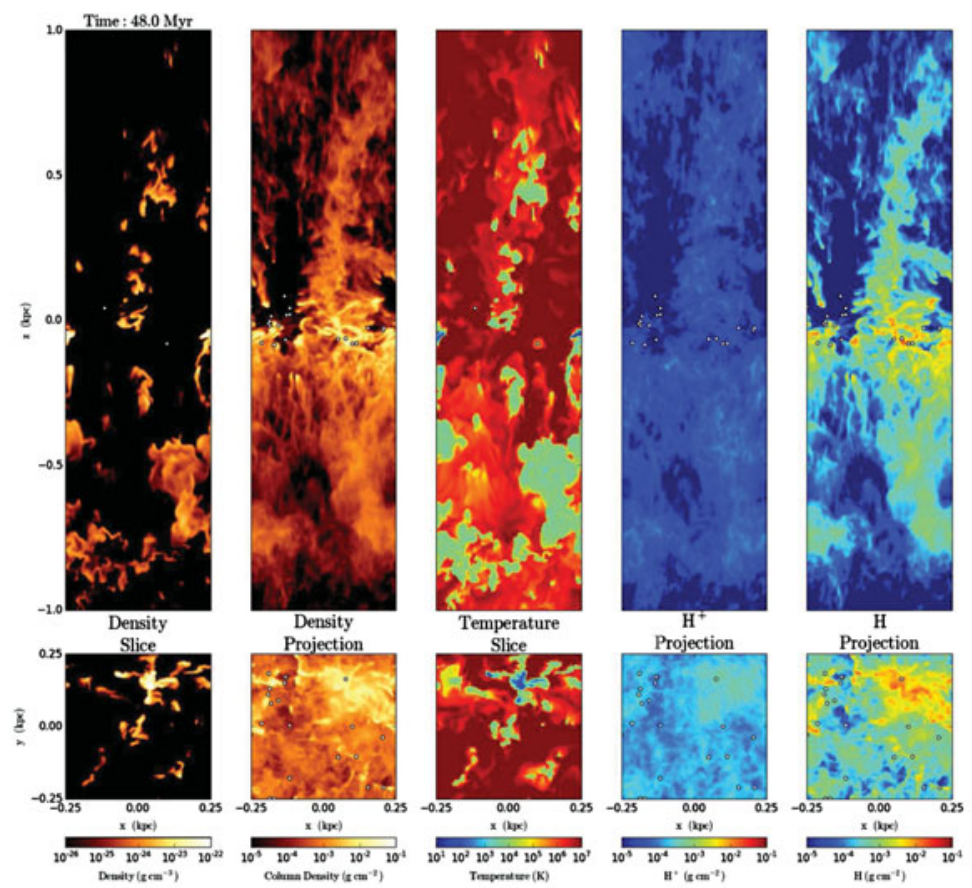

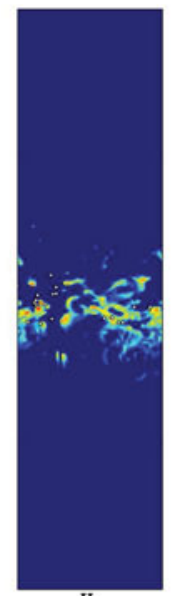

$\mathrm{H}_{2}$

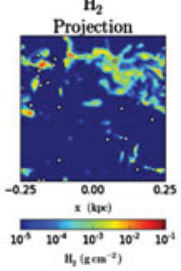

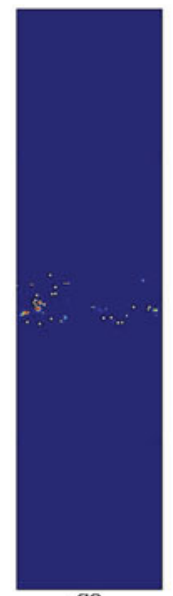

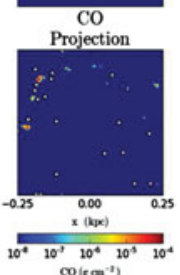

Figure 3. Top: Snapshot of a SILCC run with cluster sink particles and magnetic fields at $t=48 \mathrm{Myr}$. Only a section of the simulation box, which measures $0.5 \mathrm{kpc} \times 0.5 \mathrm{kpc} \times 2 \mathrm{kpc}$, is shown. From left to right: Gas density (slice), column density (projection), gas temperature (slice), and column densities of $\mathrm{H}^{+}, \mathrm{H}$ and $\mathrm{H}_{2}$, and $\mathrm{CO}$. All slices are taken at $y=0$ (elongated panels), or at $z=0$ (square panels), respectively. Accordingly, the column density is projected along the $y$-or $z$-axis. The winds and Supernovae from the massive stars inside the clusters are followed. Figure from Gatto et al. (in prep.). (A color version is available online.)

could be an important source of energy and momentum. However, it is unclear how quickly the hot wind would cool as this depends on the stability of the wind-blown shell (Dwarkadas et al., 2007). It seems that the indirect effect of pressure-driven winds do not have a large dynamical impact on the surrounding MCs, as shown in recent numerical studies by Dale et al. (2014). Nevertheless, the momentum input of winds could still be significant (Rogers \& Pittard, 2013; van Marle et al., 2015). In models of uniform ambient media with ionization and winds, the wind is generally trapped within the HII region and thus has a limited effect (Toalá \& Arthur, 2011; Geen et al., 2015). Overall, wind feedback might be negligible compared to the effects of ionizing radiation (Matzner, 2002).

\section{The SILCC project II: Winds and Supernovae.}

In a follow-up study (Gatto et al., in prep.), we now include self-consistent star formation, which is modelled using sink particles that represent star clusters. We follow the evolution of each individual massive star that is formed within a cluster by tracking its stellar wind feedback and its Supernova explosion. In these new models (an example is shown in Fig. 3 ), we find that stellar winds are important regulators of the star formation efficiency in these discs, as they limit the accretion onto the star-forming MCs and therefore quench the formation of gravitationally bound molecular clouds and star formation before the first Supernovae explode. 


\section{Conclusions}

There are several viable models that aim to explain the observed KS and molecular KS relations on galactic scales. New theoretical models work to incorporate changes of $n_{\mathrm{KS}}$ with Galactocentric radius and/or galaxy type. However, almost all of the models require stellar feedback to explain the low global star formation efficiency of just $\sim 1 \%$. Although Supernova feedback adds the largest momentum to the ISM, it is most likely not the dominant mechanism to limit $\epsilon_{\mathrm{SF}}$ because Supernovae explode late during the evolution of a stellar cluster and are not well correlated with the dense gaseous component. Also, a Supernova fraction in low density gas seems to be required in order to push gas into a galactic fountain or outflow. Other feedback processes are necessary to prevent star formation in the first place. Here we could demonstrate that stellar winds can limit the accretion onto young clusters and significantly reduce $\epsilon_{\mathrm{SF}}$. Future high-resolution models that include different forms of stellar feedback will most likely be able to solve the quest of what is regulating star formation on small scales.

\section{References}

Agertz, O., Kravtsov, A. V., Leitner, S. N., \& Gnedin, N. Y. 2013, ApJ, 770, 25

Agertz, O. \& Kravtsov, A. V., 2015, ApJ, 804, 18

Barnes, K. L., van Zee, L., Côté, S., \& Schade, D., 2012, ApJ, 757, 64

Bisbas, T. G., Wünsch, R., Whitworth, A. P., \& Hubber, D. A. 2009, A\&A, 497, 649

Bigiel, F., Leroy, A., Walter, F., Brinks, E., de Blok, W. J. G., Madore, B., \& Thornley, M. D., 2008, AJ, 136, 2846

Burkert, A. \& Hartmann, L., 2013, ApJ, 773, 48

Calzetti, D., Kennicutt, R. C., Engelbracht, C. W., Leitherer, C., Draine, B. T., Kewley, L., Moustakas, J., et al. , 2007, 666, 870

Chabrier, G., 2001, ApJ, 554, 1274

Clark, P. C. \& Glover, S. C. O., 2014, MNRAS, 444, 2396

Dale, J. E., Ercolano, B., \& Bonnell, I. A. 2012, MNRAS, 424, 377.

Dale, J. E., Nogoumou, J., Ercolano, B., \& Bonnell, I. A. 2014, MNRAS, 442, 694.

Dwarkadas, V. V. 2007, ApJ, 667, 226

Elmegreen, B., 1997, $R M x A C, 6,165$

Elmegreen, B. G. \& Hunter, D. A., 2015, ApJ, 805, 145

Elmegreen, B. G., 2015, ApJ, 814, 30

Evans, I. I., N. J. et al. , 2009, ApJS, 181, 321

Evans, I. I., N. J., Heiderman, A., \& Vutisalchavakul, N., 2014, ApJ, 782, 114

Fall, S. M., Krumholz, M. R., \& Matzner, C. D. 2011, ApJ, 710, 142

Federrath, C. \& Klessen, R. S., 2013, ApJ, 763, 51

Fryxell, B., Olson, K., Ricker, P., \& Timmes, et al. 2000, ApJS, 131, 273

Gatto, A., Walch, S., MacLow, M.-M., \& Naab, T., et al. 2015, MNRAS, 449, 1057

Gatto, A., Walch, S., Girichidis, P., et al. in preparation

Geen, S., Rosdahl, J., Blaizot, J., Devriendt, J., \& Slyz, A. 2015, MNRAS, 448, 3248

Gent, F. A., Shukurov, A., Fletcher, A., Sarson, G. R., \& Mantere, M. J., 2013, MNRAS, 432, 1396

Genzel, R., Tacconi, L. J., Gracia-Carpio, J., Sternberg, A., Cooper, M. C., Shapiro, K., Bolatto, A., Bouché, N., et al. , 2010, MNRAS, 407, 2091

Genzel, R., Tacconi, L. J., Lutz, D., Saintonge, A., Berta, S., Magnelli, B., Combes, F., GarcíaBurillo, S., et al. 2015, ApJ, 800, 20

Girichidis, P., Walch, S., Naab, et al. 2016, MNRAS, 436, 3432

Glover, S. C. O. \& Clark, P. C., 2012, MNRAS, 421, 9

Harfst, S., Theis, Ch., \& Hensler, G., 2006, A\& $A$, 449, 509

Heiderman, A., Evans, N. J., II, Allen, L. E., Huard, T., \& Heyer, M., 2010, ApJ, 723, 1019 
Hill, A. S., Joung, M. R., Mac Low, M.-M., Benjamin, R. A., Haffner, L. M., Klingenberg, C., \& Waagan, K., 2012, ApJ, 750, 104

Joung, M. K. R. \& Mac Low, M.-M., 2006, ApJ, 653, 1266

Kennicutt, Jr., R. C., 1989, ApJ, 344, 685

Kennicutt, Jr., R. C., 1998, ApJ, 498, 541

Kim, C.-G., Kim, W.-T., Ostriker, E. C, ApJ, 743, 25

Kim, C.-G., Ostriker, E. C, Kim, W.-T., ApJ, 776, 1

Kim, C. \& Ostriker, E. C. 2015, ApJ, 802, 99

Kruijssen, J. M. D. \& Longmore, S. N., 2014, MNRAS, 439, 3239

Krumholz, M. R. \& McKee, C. F., 2005, ApJ, 630, 250

Krumholz, M. R. \& Tan, J. C., 2007, ApJ, 654, 304

Krumholz, M. R., McKee, C. F., \& Tumlinson, J., 2008, ApJ, 689, 865

Krumholz, M. R. \& Matzner, C. D. 2009, ApJ, 703, 1352

Lada, C. J., Lombardi, M., \& Alves, J. F., 2010, ApJ, 724, 687

Lada, C. J., Forbrich, J., Lombardi, M., \& Alves, J. F., 2012, ApJ, 745, 190

Leitherer, C., Schaerer, D., Goldader, J. D., González Delgado, R. M., Robert, C., Kune, D. F., de Mello, D. F., Devost, D., et al. , 1999, ApJS, 123, 3

Leroy, A. K., Walter, F., Brinks, E., Bigiel, F., de Blok, W. J. G., Madore, B., \& Thornley, M. D., 2008, AJ, 136, 2782

Matzner, Christopher D. 2002, ApJ, 566, 302

Mac Low, M.-M., Smith, M. D., Klessen, R. S., \& Burkert, A., 1998, ApSS, 261, 195

Momose, R., Koda, J., Kennicutt, R. C., Jr., Egusa, F. Calzetti, D., Liu, G., Donovan Meyer, J., \& Okumura, S. K., 2013, ApJ, 772, 13

Murray, N., 2011, ApJ, 729, 133

Onodera, S., Kuno, N., Tosaki, T., Kohno, K., Nakanishi, K., Sawada, T., Muraoka, K., Komugi, S., et al. , 2010, ApJ, 722, 127

McKee, C. F. \& Ostriker, J. P. 1977, ApJ, 218, 148

Ostriker, E. C., McKee, C. F., \& Leroy, A. K., 2010, ApJ, 721, 975

Padoan, P., Nordlund, A., \& Jones, B. J. T., 1997, MNRAS, 288, 145

Padoan, P., Nordlund, A., ApJ, 576, 870

Pringle, J. E., Allen, R. J., \& Lubow, S. H., 2001, MNRAS, 327, 663

Murray, N., Quataert, E., \& Thompson, T. A. 2010, ApJ, 709, 191

Rogers, H., \& Pittard, J. M., MNRAS, 431, 1337

Roychowdhury, S., Huang, M.-L., Kauffmann, G., Wang, J., Chengalur, J. N., it MNRAS, 2015, 449,3700

Schmidt, M. 1959, ApJ, 129, 243

Schruba, A., Leroy, A. K., Walter, F., Sandstrom, K., \& Rosolowsky, E., 2010, 722, 1699

Schruba, A., Leroy, A. K., Walter, F., Bigiel, F., Brinks, E., de Blok, W. J. G., Dumas, G., Kramer, C., Rosolowsky, E., et al. , 2011, 142, 37

Salpeter, E. E., 1955, ApJ, 121, 161

Shetty, R., Kelly, B. C., Rahman, N., Bigiel, F., Bolatto, A. D., Clark, P. C., Klessen, R. S., \& Konstandin, L. K., 2014, 437, 61

Shetty, R., Clark, P. C., \& Klessen, R. S., 2014, 442, 2208

Silk, J., 1997, ApJ, 481, 703

Sternberg, A., Le Petit, F., Roueff, E., \& Le Bourlot, J., 2014, ApJ, 790, 10

Stone, J. M., Ostriker, E. C., \& Gammie, C. F., 1998, it ApJL, 508, 99

Tacconi, L. J., Neri, R., Genzel, R., Combes, F., Bolatto, A., Cooper, M. C., Wuyts, S., Bournaud, F., et al. ,2013, ApJ, 768, 74

\&Tasker, E. J.; Bryan, G. L., 2006, ApJ, 641, 878

Toalá, J. A. \& Arthur, S. J. 2011, ApJ, 737, 100

van Marle, A. J., Meliani, Z., \& Marcowith, A. 2015, A\& A, 584, 49

Vazquez-Semadeni, E., 1994, ApJ, 423, 681

Wada, K. \& Norman, C. A., 2007, ApJ, 660, 276

Walch, S.,Whitworth, A. P., Bisbas, T., Wünsch, R., Hubber, D. 2012, MNRAS, 427, 625

Walch, S., Girichidis, P., Naab, T., et al. 2015, MNRAS, 454, 238 